\title{
Asymptotic analysis of wall modes in a flexible tube
}

\author{
V. Kumaran ${ }^{\mathrm{a}}$ \\ Department of Chemical Engineering, Indian Institute of Science, Bangalore 560 012, India
}

\begin{abstract}
The stability of wall modes in a flexible tube of radius $R$ surrounded by a viscoelastic material in the region $R<r<H R$ in the high Reynolds number limit is studied using asymptotic techniques. The fluid is a Newtonian fluid, while the wall material is modeled as an incompressible visco-elastic solid. In the limit of high Reynolds number, the vorticity of the wall modes is confined to a region of thickness $O\left(\epsilon^{1 / 3}\right)$ in the fluid near the wall of the tube, where the small parameter $\epsilon=R e^{-1}$, and the Reynolds number is $R e=(\rho V R / \eta), \rho$ and $\eta$ are the fluid density and viscosity, and $V$ is the maximum fluid velocity. The regime $\Lambda=\epsilon^{-1 / 3}\left(G / \rho V^{2}\right) \sim 1$ is considered in the asymptotic analysis, where $G$ is the shear modulus of the wall material. In this limit, the ratio of the normal stress and normal displacement in the wall, $\left(-\Lambda C\left(k^{*}, H\right)\right)$, is only a function of $H$ and scaled wave number $k^{*}=(k R)$. There are multiple solutions for the growth rate which depend on the parameter $\Lambda^{*}=k^{* 1 / 3} C\left(k^{*}, H\right) \Lambda$. In the limit $\Lambda^{*} \ll 1$, which is equivalent to using a zero normal stress boundary condition for the fluid, all the roots have negative real parts, indicating that the wall modes are stable. In the limit $\Lambda^{*} \gg 1$, which corresponds to the flow in a rigid tube, the stable roots of previous studies on the flow in a rigid tube are recovered. In addition, there is one root in the limit $\Lambda^{*} \gg 1$ which does not reduce to any of the rigid tube solutions determined previously. The decay rate of this solution decreases proportional to $\left(\Lambda^{*}\right)^{-1 / 2}$ in the limit $\Lambda^{*} \gg 1$, and the frequency increases proportional to $\Lambda^{*}$.
\end{abstract}

PACS. 83.50.-v Deformation; material flow - 47.15.Fe Stability of laminar flows - 47.60.+i Flows in ducts, channels, nozzles, and conduits

\section{Introduction}

Many biological systems and biotechnology processes involve flow through flexible tubes and channels. The flow of blood and other fluids in the body takes place through flexible tubes, and the separation and purification processes in pharmaceutical industries often involve flow in tubes and channels made up of polymer matrices and membranes. These have been analyzed using models similar to those for the flow in a rigid tube, but some experiments conducted by Krindel and Silberberg [1] suggest that the characteristics of the flow in a flexible tube could be very different. The drag force in a flexible tube is much larger than that in a rigid tube of the same radius at Reynolds numbers where the flow in a rigid tube is laminar, and the anomalous drag force could not be accounted for by changes in the radius of the tube. This led them to conclude that the Reynolds number at which the flow changes from the laminar to turbulent regime is much smaller than the transition Reynolds number $R e=2300-4000$ for a rigid tube, and the transition Reynolds number is influenced by the elasticity of the surface. This instability is qualitatively different from

\footnotetext{
a e-mail: kumaran@chemeng.iisc.ernet.in
}

the "pearling" instability observed in cylindrical vesicles $[2,3]$, because the instability is induced by fluid flow, in contrast to the instability of a cylindrical fluid membrane due to external excitation. It is useful, from a technological viewpoint, to develop a fundamental understanding of the factors affecting this transition, since this would help in optimizing the design of biotechnological processes. In applications where it is important to have high mass or heat transfer rates, it would be necessary to operate the system in the turbulent regime where the transfer rates are up to three orders of magnitude higher than that in the laminar regime. In processes where low drag forces are desirable, the system could be operated in the laminar regime.

At the point of transition from the laminar to turbulent regime, the laminar flow becomes unstable to small perturbations, and so it is necessary to study the stability characteristics of the flow to predict the Reynolds number at which there is a transition from stable to unstable modes. The stability of the "viscous modes" in the flow through a flexible channel in the low Reynolds number regime, where $R e \equiv(\rho V R / \eta) \ll 1$, and $(V \eta / G R) \sim 1$ was analyzed by Kumaran, Fredrickson and Pincus [4]. Here, $\rho$ and $\eta$ are the density and viscosity of the fluid, $R$ is the channel thickness or tube radius, $V$ is the characteristic fluid velocity and $G$ is the shear modulus of the wall 
material. In this regime, there is a balance between the viscous forces in the fluid and the elastic forces in the wall. It was observed that the viscous modes become unstable when the fluid velocity is increased beyond a critical value. The analysis was extended to the flow in a flexible tube by Kumaran [5]. The stability of the "inviscid modes" in the high Reynolds number regime $R e \gg 1$ and $\left(\rho V^{2} / G\right) \sim 1$, was analyzed using asymptotic techniques for a channel flow [6] and for a tube flow [7]. In this case, there is a balance between the inertial forces in the fluid and the elastic forces in the wall, and the analysis indicated that the inviscid modes are always stable.

The above asymptotic results are rather paradoxical, because they predict that the flow in the low Reynolds number regime could become unstable, while the flow in the high Reynolds number regime is always stable. In order to resolve this paradox, a numerical continuation of the unstable viscous modes into the intermediate Reynolds number regime was undertaken by Srivatsan and Kumaran [8] for the flow in a flexible channel, and by Kumaran [9] for the flow in a flexible tube. In these studies, it was found that the instability does persist into the intermediate Reynolds number regime, but the critical Reynolds number is much larger than the Reynolds number at which the inviscid modes are observed for a given set of fluid and wall properties. In addition, a boundary layer of thickness $O\left(R e^{-1 / 3}\right)$ smaller than the width of the channel thickness or tube radius was observed for these unstable modes in the high Reynolds number regime. This is very different from the boundary layer of thickness $O\left(R e^{-1 / 2}\right)$ for the inviscid modes. The $O\left(R e^{-1 / 3}\right)$ scaling is characteristic of a set of modes called "wall modes". The purpose of the present analysis is to examine these wall modes in the high Reynolds number regime using asymptotic analysis. The analysis is carried out for a flexible tube, because results are available for a rigid tube in the high Reynolds number regime, and this facilitates validation of the present results. However, the results could easily be extended to the flow in a channel with flexible walls.

There are two types of high Reynolds number modes in a rigid tube where the vorticity is confined to thin regions near the center of the tube or at the wall [10]. The vorticity of the "center modes" is confined to a region of thickness $O\left(R e^{-1 / 4}\right)$ at the center of the tube, and the decay rate of these modes is $O\left(R e^{-1 / 2}\right)$ smaller than the fluid strain rate. The vorticity of the "wall modes" is confined to a layer of thickness $O\left(R e^{-1 / 3}\right)$ at the wall, and the damping rate of these modes is $O\left(R e^{-1 / 3}\right)$ smaller than the fluid strain rate. The flexibility of the tube will not affect the center modes because the vorticity is confined to the center, but the wall modes could be affected due to the wall flexibility. It should be noted that the wall mode is distinct from the "inviscid mode" for a flexible tube which was analyzed earlier [7], and the differences are summarized in Section 3.

The stability characteristics are first determined using asymptotic analysis in the high Reynolds number limit in Section 2. A scaling analysis shows that the elastic stresses

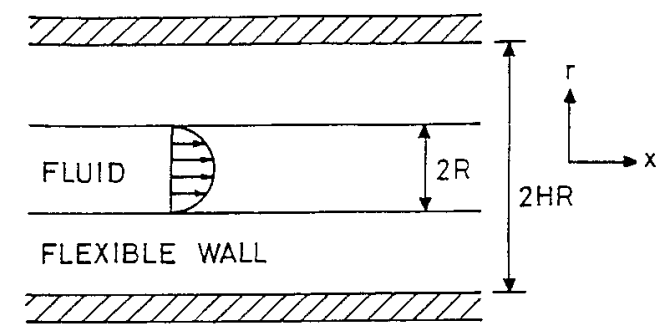

Fig. 1. Configuration and definition of coordinate systems.

in the wall affect the damping of the wall modes when the dimensionless number $\Lambda \equiv \epsilon^{-1 / 3}\left(G / \rho V^{2}\right)^{1 / 2} \sim 1$, where $\epsilon \equiv R e^{-1}$. It is shown, using scaling arguments, that the inertial and viscous stresses are of the same magnitude in the wall layer, and the parameter $\Lambda$ represents the ratio of the elastic stresses in the wall material and the inertial (or viscous) stresses in the fluid. The velocity and stress fields are expanded in the small parameter $\epsilon$, and an asymptotic analysis is used to determine the growth rate of the wall modes. In the regime $\Lambda \sim 1$, the elastic stress is large compared to the inertial stress in the wall material, and the wall admittance is only a function of the ratio of radii of the wall and the fluid $H$ and the wave number $k$ of the perturbations. There are multiple solutions for the growth rate of the perturbations. In the limit $\Lambda \rightarrow \infty$, the solutions reported previously for a rigid tube are recovered. In addition, there is one mode in a flexible tube whose growth rate does not converge to any of the rigid tube modes, but which has a diverging frequency in the limit $\Lambda \rightarrow \infty$. This is the least stable wall mode in a flexible tube, and its decay rate decreases proportional to $\Lambda^{-1 / 2}$ in the limit $\Lambda \rightarrow \infty$.

\section{Analysis}

The configuration consists of a tube of radius $R$ surrounded by a visco-elastic solid in the region $R<r<H R$ as shown in Figure 1. The mean flow in the tube is a steady Hagen-Poiseuille flow:

$$
\bar{v}_{i}=V\left(1-\frac{r^{2}}{R^{2}}\right) \delta_{i x}
$$

where $V$ is the maximum velocity at the center of the tube. In the linear stability analysis, a small perturbation is added to the mean velocity of the form $v_{i}=\bar{v}_{i}+v_{i}^{\prime}$, and the growth rate of the perturbation is determined. The dynamical equations for the velocity perturbation are the linearized Navier-Stokes equations for an incompressible Newtonian fluid:

$$
\begin{gathered}
\partial_{i} v_{i}^{\prime}=0 \\
\rho\left[\partial_{t} v_{i}^{\prime}+\left(\bar{v}_{j} \partial_{j} v_{i}^{\prime}+v_{j}^{\prime} \partial_{j} \bar{v}_{i}\right)\right]=-\partial_{i} p^{\prime}+\eta \partial_{j}^{2} v_{i}^{\prime}
\end{gathered}
$$

where $\partial_{t} \equiv(\partial / \partial t), \partial_{i} \equiv\left(\partial / \partial x_{i}\right), \rho$ and $\eta$ are the density 
and viscosity of the fluid and $p^{\prime}$ is the pressure perturbation. The stress due to the perturbation in the velocity field is:

$$
\tau_{i j}^{\prime}=-p^{\prime} \delta_{i j}+\eta\left(\partial_{i} v_{j}^{\prime}+\partial_{j} v_{i}^{\prime}\right)
$$

The wall of the tube is made up of a visco-elastic material whose dynamics is described by a displacement field $u_{i}^{\prime}$ which represents the displacement of material points from their steady state positions due to the stresses at the surface. The dynamical equations for the displacement field $u_{i}^{\prime}$ are similar to those used for incompressible elastic solids (Landau and Lifshitz [11]) modified to include viscous effects. Similar equations have been used for the study of surface fluctuations in polymer gels (Harden, Pleiner and Pincus [12], Kumaran [13]) and in the earlier studies of the instability in flexible tubes (Kumaran $[5-7,9]$ ). The displacement field for the incompressible visco-elastic material satisfies the solenoidal condition:

$$
\partial_{i} u_{i}^{\prime}=0
$$

while the momentum balance equation is:

$$
\rho \partial_{t}^{2} u_{i}^{\prime}=-\partial_{i} p^{\prime}+G \partial_{j}^{2} u_{i}^{\prime}+\eta_{s} \partial_{j}^{2} v_{i}^{\prime}
$$

where $G$ is the shear modulus, $\eta_{s}$ is the viscosity of the visco-elastic material that comprises the surface and the velocity $v_{i}^{\prime}=\partial_{t} u_{i}^{\prime}$. For simplicity, it has been assumed that the density of the wall material is the same as that of the fluid. The first term on the right side of the above expression is the gradient of the pressure required to enforce incompressibility, the second is the divergence of an elastic stress due to the strain in the medium and the third is the divergence of a viscous stress due to the strain rate. The stress tensor in the wall is:

$$
\sigma_{i j}^{\prime}=-p^{\prime} \delta_{i j}+G\left(\partial_{i} u_{j}^{\prime}+\partial_{j} u_{i}^{\prime}\right)+\eta_{s}\left(\partial_{i} v_{j}^{\prime}+\partial_{j} v_{i}^{\prime}\right) .
$$

In the constitutive relation (7), it is assumed that the shear modulus $G$ and the viscosity of the wall $\eta_{s}$ are independent of the frequency. The validity of these assumptions was discussed in detail in Kumaran [9]. The assumption regarding the shear modulus is valid over a wide range of frequencies for polymer gels, and the value of the modulus is referred to as the plateau modulus. The loss modulus of polymer gels does not exhibit the same property, and so the assumption regarding the viscosity may not be valid for polymer gels. However, the results of the present analysis can easily be adapted to situations where the viscosity is frequency dependent, if the frequency dependence of the viscosity is known.

The boundary conditions at the center of the tube are $v_{r}^{\prime}=0$ and $\partial_{r} v_{x}^{\prime}=0$ due to axisymmetry. In addition, it is necessary to specify boundary conditions at the outer surface $r=H R$ for the displacement field in the wall material. For definiteness, we use zero displacement conditions $u_{r}^{\prime}=0$ and $u_{x}^{\prime}=0$ at this surface. However, it should be noted that the boundary conditions only affect the constant $C\left(k^{*}, H\right)$ in equation (31), which gives the ratio of the normal velocity and normal stress at the interface. Though there is a quantitative variation in this ratio if a different boundary condition such as the zero stress condition is used at the surface $r=H R$, the qualitative nature of the wall modes remain unchanged. The boundary conditions at the interface between the fluid and the wall are the continuity of velocity and stress applied at the perturbed interface between the fluid and the wall. It is convenient to expand the velocity and stress fields at the perturbed interface in a Taylor series about their values at the unperturbed interface $r=R$. The linear terms in the expansion are retained and the higher order terms neglected to obtain the following conditions where all the quantities are evaluated at the unperturbed interface $r=R$ :

$$
\begin{aligned}
v_{r}^{\prime} & =\partial_{t} u_{r}^{\prime}, \\
v_{x}^{\prime}+u_{r}^{\prime} \partial_{r} \bar{v}_{x} & =\partial_{t} u_{x}^{\prime}, \\
\tau_{r r}^{\prime} & =\sigma_{r r}^{\prime}, \\
\tau_{x r}^{\prime} & =\sigma_{x r}^{\prime} .
\end{aligned}
$$

In the boundary condition for the axial velocity, the additional term on the left side is the variation in the mean velocity at the surface due to a change in the surface height.

In the linear stability analysis, the perturbations to the velocity and displacement fields are expressed as the sum of Fourier modes periodic in the $x$ direction,

$$
\begin{aligned}
v_{i}^{\prime} & =\tilde{v}_{i}(r) \exp (i k x+s t), \\
u_{i}^{\prime} & =\tilde{u}_{i}(r) \exp (i k x+s t),
\end{aligned}
$$

where $k$ is the wave number and $s$ is the growth rate of the perturbations. It is useful to discuss the derivation for the length, time and velocity scales for the wall modes. The linearized Navier-Stokes equations for the Fourier modes (9) are

$$
\begin{gathered}
\left(d_{r}+r^{-1}\right) \tilde{v}_{r}+i k \tilde{v}_{x}=0 \\
\rho\left(s+i k V\left(1-(r / R)^{2}\right)\right) \tilde{v}_{r}=-d_{r} \tilde{p} \\
+\eta\left(d_{r}^{2}+r^{-1} d_{r}-r^{-2}-k^{2}\right) \tilde{v}_{r} \\
\left.\rho\left(s+i k V\left(1-(r / R)^{2}\right)\right)\right) \tilde{v}_{x}+\rho \tilde{v}_{r}\left(-2 V r / R^{2}\right)=-i k \tilde{p} \\
+\eta\left(d_{r}^{2}+r^{-1} d_{r}-k^{2}\right) \tilde{v}_{x}
\end{gathered}
$$

where (1) has been inserted for the mean velocity $\bar{v}_{i}$. In the high Reynolds number limit, the inertial effects are large compared to viscous effects, and one could attempt to obtain a leading order solution by neglecting viscous effects. The inviscid equations in this limit are

$$
\begin{gathered}
\left(d_{r}+r^{-1}\right) \tilde{v}_{r}+i k \tilde{v}_{x}=0 \\
\rho\left(s+i k V\left(1-(r / R)^{2}\right)\right) \tilde{v}_{r}+d_{r} \tilde{p}=0 \\
\rho\left(s+i k V\left(1-(r / R)^{2}\right)\right) \tilde{v}_{x}+\rho \tilde{v}_{r}\left(-2 V r / R^{2}\right)+i k \tilde{p}=0 .
\end{gathered}
$$


However, the viscous terms in the conservation equation contain the highest derivatives, and the neglect of these terms converts the momentum equations from second order to first order differential equations. Consequently, it is not possible to satisfy all the velocity and stress boundary conditions required for the original viscous second order differential equations, and only the normal velocity and normal stress conditions can be satisfied at the interface between the fluid and the wall material. To satisfy the tangential velocity and stress boundary conditions at the wall, it is necessary to postulate a viscous "wall layer" of thickness $\delta R$ where $\delta \ll 1$ where the viscous effects are significant. Consequently, it is useful to define an "outer flow" with velocity $\tilde{v}_{o i}$ in the core of the tube, where the distance from the wall is large compared to $O(R)$, and the inviscid equations $(13,14,15)$ are applicable, and a "wall layer" with velocity $\tilde{v}_{w i}$ where the distance from the wall is $O(\delta R)$ and the viscous effects are significant. The parameter $\delta$ is determined by balancing the viscous and inertial terms in the conservation equations in the wall layer.

In the wall layer, a scaled distance from the wall $z^{*}$ is defined as $z^{*}=(1-r / R) / \delta$ with $\delta \ll 1$. The linearized Navier-Stokes equations (10-12), expressed in terms of this scaled coordinate, are

$$
\begin{aligned}
& \left(-(R \delta)^{-1} d_{z^{*}}+R^{-1}\left(1-z^{*} \delta\right)^{-1}\right) \tilde{v}_{w r}+i k \tilde{v}_{w x}=0 \\
& \rho\left(s+i k V\left(1-(r / R)^{2}\right)\right) \tilde{v}_{w r}=(R \delta)^{-1} d_{z^{*}} \tilde{p}_{w} \\
& \quad+\eta\left((R \delta)^{-2} d_{z^{*}}^{2}-(R \delta)^{-1} R^{-1}\left(1-\delta z^{*}\right)^{-1} d_{z^{*}}\right. \\
& \left.\quad+R^{-2}\left(1-\delta z^{*}\right)^{-2}-k^{2}\right) \tilde{v}_{w r}
\end{aligned}
$$

$$
\begin{aligned}
& \rho\left(s+i k V\left(1-(r / R)^{2}\right)\right) \tilde{v}_{w x}+\rho \tilde{v}_{w r}\left(-2 V r / R^{2}\right)=-i k \tilde{p}_{w} \\
& +\eta\left((R \delta)^{-2} d_{z^{*}}^{2}-(R \delta)^{-1} R^{-1}\left(1-\delta z^{*}\right)^{-1} d_{z^{*}}-k^{2}\right) \tilde{v}_{w x}
\end{aligned}
$$

where $\tilde{v}_{w r}$ and $\tilde{v}_{w x}$ are the velocities in the wall layer. From equation (16), it can be inferred that $\tilde{v}_{w x} \sim \delta^{-1} \tilde{v}_{w r}$ for a balance to be achieved between the two terms on the left side (it is assumed that the wave number $\left.k \sim R^{-1}\right)$. It is useful to define a scaled axial velocity $\tilde{v}_{w x}^{*}=\left(\tilde{v}_{w x} / V\right)$, and the appropriate scaled radial velocity is $\tilde{v}_{w r}^{*}=\left(\tilde{v}_{w r} / \delta V\right)$. With this definition, the mass conservation equation correct to leading order in small $\delta$ is

$$
-d_{z^{*}} \tilde{v}_{w r}^{*}+i k^{*} \tilde{v}_{w x}^{*}=0
$$

where the scaled wave number $k^{*}=(k R)$. The leading order momentum conservation equation in the $r$ direction, expressed in terms of $\tilde{v}_{w r}^{*}$ and $\tilde{v}_{w x}^{*}$, is

$$
\begin{aligned}
\rho(s+ & \left.2 i k^{*}(V / R) \delta z^{*}\right) V \tilde{v}_{w x}^{*}-2 \rho\left(V^{2} / R\right) \delta \tilde{v}_{w r}^{*}= \\
& -i k^{*} \tilde{p}_{w} / R+\left(\eta / R^{2}\right) \delta^{-2} d_{z^{*}}^{2}\left(V \tilde{v}_{w x}^{*}\right)
\end{aligned}
$$

In equation (20), only the leading order term in the expression for the mean velocity $\bar{v}_{x}$ has been retained, and the higher order terms have been neglected. A balance between the inertial and viscous terms in the above equation can be achieved in two ways. The first is to consider the case $\delta \sim\left(\eta / \rho R^{2} s\right)^{1 / 2}$. This corresponds to the "inviscid modes" analyzed earlier by the author [6,7]. In this case, the growth rate $s$ is is determined from the inviscid equations $(13,14,15)$, and this is inserted into the scaled equations for the wall layer in order to determine the velocity profile in the wall layer. It can be easily verified from $(13,14,15)$ that the growth rate for the inviscid modes scales as $s \sim(V / R)$, and consequently the boundary layer thickness scales as $\delta \sim R e^{-1 / 2}$. Another way to achieve a balance between the inertial and viscous terms in (20) is to consider the case $s \sim(\delta V / R)$, and $(\rho \delta V / R) \sim\left(\eta / R^{2} \delta^{2}\right)$. This balance indicates that the boundary layer thickness scales as $\delta \sim R e^{-1 / 3}$, and the growth rate scales as $s \sim R e^{-1 / 3}(V / R)$. This balance corresponds to the "wall modes". Without loss of generality, the small parameter $\delta$ is defined as $\delta=R e^{-1 / 3}$, and a scaled growth rate $s^{*}$ is defined as $s^{*}=\left(R e^{1 / 3} s R / V\right)$, to give the following equation

$$
\left(s^{*}+2 i k^{*} z^{*}\right) \tilde{v}_{w x}^{*}-2 \tilde{v}_{w r}^{*}=-i k^{*} \tilde{p}_{w}^{*}+d_{z^{*}}^{2} \tilde{v}_{w x}^{*}
$$

where the scaled pressure $\tilde{p}_{w}^{*}=\left(R e^{1 / 3} \tilde{p}_{w} /\left(\rho V^{2}\right)\right)$. The momentum conservation equation in the $r$ direction (17) is simplified using the scaled expressions for the velocities, pressure and the growth rate, and the leading order terms in the small parameter $\delta$ are retained to obtain the following leading order scaled equation

$$
d_{z^{*}} \tilde{p}_{w}^{*}=0
$$

From (22), it is seen that the variation of the pressure in the direction normal to the flow is zero in the leading approximation; this is the common feature of boundary layer flows. The solution of the equations $(19,21,22)$ are provided in Appendix A, and the results are

$$
\begin{aligned}
\tilde{v}_{w x}^{*} & =A i(y, 1), \\
\tilde{v}_{w r}^{*} & =2^{-1 / 3}\left(i k^{*}\right)^{2 / 3}[y A i(y, 1)-A i(y,-1)], \\
\tilde{p}_{w}^{*} & =0 .
\end{aligned}
$$

where $A i(y, p)$ are generalized Airy functions of order $p$ defined in Appendix A, and the argument $y$ is

$$
y=\left(2 i k^{*}\right)^{1 / 3}\left[\left(s^{*} / 2 i k^{*}\right)+z^{*}\right] .
$$

The solution (23) indicates that the $O\left(R e^{-1 / 3} \rho V^{2}\right)$ contribution to the pressure in the wall layer is zero. To apply the normal stress continuity at the interface, it is necessary to determine the largest non-zero contribution to the pressure in the wall layer. This can be obtained by balancing the pressure and inertial terms in the momentum conservation equation (17), and it is easily inferred that the pressure scales as $\tilde{p}_{w} \sim R e^{-1}(\rho V) \tilde{v}_{w x}$. For future reference, the magnitudes of the velocity and stress fields in 
the wall layer are:

$$
\begin{aligned}
\tilde{v}_{w r} & \sim \epsilon^{1 / 3} \tilde{v}_{w x} \\
\tilde{\tau}_{w r r} & \sim \epsilon(\rho V) \tilde{v}_{w x} \\
\tilde{\tau}_{w x r} & \sim \epsilon^{2 / 3}(\rho V) \tilde{v}_{w x}
\end{aligned}
$$

where $\epsilon=R e^{-1}$ is a small parameter. In the above equation, the normal stress is of the same magnitude as the pressure, $\tilde{p}_{w}$, while the shear stress scales as $\eta d_{r} \tilde{v}_{w x}$, which is $O\left(\epsilon^{2 / 3} \rho V \tilde{v}_{w x}\right)$, since $d_{r} \tilde{v}_{w x} \sim \epsilon^{-1 / 3}\left(\tilde{v}_{w x} / R\right)$.

The equations for the inviscid outer flow in the tube are $(13,14,15)$. It can be shown, as follows, that the boundary conditions at the interface, (Eq. (8)), imply that the velocities in the outer region are $O\left(\epsilon^{1 / 3}\right)$ smaller than the tangential velocity in the wall layer $\tilde{v}_{w x}$. In the wall material, the radial and tangential displacement fields are of the same magnitude. From the tangential velocity boundary condition in (8), the displacement $\tilde{u}_{r} \sim(R / V) \tilde{v}_{w x}$ where $\tilde{v}_{w x}$ is the magnitude of the tangential velocity in the wall layer. Inserting this into the normal velocity boundary condition in (8), it can be seen that the velocity field in the outer flow $\tilde{v}_{\text {or }} \sim \epsilon^{1 / 3} \tilde{v}_{w x}$. In addition, $\tilde{v}_{o x} \sim \epsilon^{1 / 3} \tilde{v}_{w x}$ because the radial and tangential velocities are of the same magnitude in the outer region. From (15), it can be verified that the pressure is $O\left(\epsilon^{1 / 3} \rho V \tilde{v}_{w x}\right)$. The magnitudes of the velocity and the stress fields in the outer flow, similar to (25) for the wall layer, are:

$$
\begin{aligned}
\tilde{v}_{o r} & \sim \epsilon^{1 / 3} \tilde{v}_{w x}, \\
\tilde{v}_{o x} & \sim \epsilon^{1 / 3} \tilde{v}_{w x}, \\
\tilde{\tau}_{o r r} & \sim \epsilon^{1 / 3}(\rho V) \tilde{v}_{w x}, \\
\tilde{\tau}_{o x r} & \sim \epsilon(\rho V) \tilde{v}_{w x} .
\end{aligned}
$$

In $(26)$, the normal stress $\tilde{\tau}_{\text {orr }}$ has the same magnitude as the pressure $\tilde{p}_{o}$ in the outer region. For the subsequent analysis, it is not necessary to obtain detailed solutions for equations $(13,14,15)$ for the outer flow, but it is sufficient to use a scaling analysis to obtain the relation between the pressure and the radial velocity at the wall. Using the scaled velocity $\tilde{v}_{o i}^{*}=\left(\tilde{v}_{o i} / \epsilon^{1 / 3} V\right)$ and scaled pressure $\tilde{p}_{o}^{*}=\left(\epsilon^{-1 / 3} \tilde{p}_{o} / \rho V^{2}\right)$, equation (15) has the following form at the wall $r=R$ in the leading approximation:

$$
\tilde{p}_{o}^{*}=2\left(i k^{*}\right)^{-1} \tilde{v}_{o r}^{*}
$$

This provides a relation between the pressure and the normal velocity due to the outer flow at the wall. Note that the term $\rho s \tilde{v}_{w x}$ in equation (15) has been neglected, because it is $O\left(\epsilon^{1 / 3}\right)$ smaller than the terms retained above.

The equations for the displacement field in the wall material are obtained using the form (8) for the displacement field. The details of the calculation for the eigenfunctions $\tilde{u}_{i}(r)$ are provided in Appendix B. It is sufficient for the present purposes to discuss the magnitudes of the displacement and stress fields in the wall materials. From the boundary condition for the tangential velocity (8), it can be seen that $\tilde{u}_{r} \sim\left(\tilde{v}_{w x} R / V\right)$. The normal stress in the fluid scales as $\tilde{\tau}_{\text {orr }} \sim \epsilon^{1 / 3} \rho V \tilde{v}_{w x}$ from (26), while the elastic stress in the wall scales as $\tilde{\sigma}_{r r} \sim\left(G \tilde{u}_{r} / R\right)$. The dynamics of the wall material will influence the fluid flow only if these two stresses are of the same magnitude, which requires that the dimensionless number $\Lambda \equiv \epsilon^{-1 / 3}\left(G / \rho V^{2}\right) \sim 1$. With this, the magnitudes of the displacement and stress fields are:

$$
\begin{aligned}
\tilde{u}_{r} & \sim \frac{R \tilde{v}_{w x}}{V}, \\
\tilde{u}_{x} & \sim \frac{R \tilde{v}_{w x}}{V}, \\
\tilde{\sigma}_{r r} & \sim \Lambda\left(\epsilon^{1 / 3} \rho V\right) \tilde{v}_{w x}, \\
\tilde{\sigma}_{x r} & \sim \Lambda\left(\epsilon^{1 / 3} \rho V\right) \tilde{v}_{w x} .
\end{aligned}
$$

The solutions for the scaled eigenfunctions $\tilde{u}_{r}^{*}=\left(\tilde{u}_{r} / R\right)$ and $\tilde{u}_{x}^{*}=\left(\tilde{u}_{x} / R\right)$ are determined in the Appendix B, and the leading order shear and normal stress fields, $\tilde{\sigma}_{r r}^{*}=$ $\tilde{\sigma}_{r r} /\left(\epsilon^{1 / 3} \rho V^{2}\right)$ and $\tilde{\sigma}_{x r}^{*}=\tilde{\sigma}_{x r} /\left(\epsilon^{1 / 3} \rho V^{2}\right)$, are:

$$
\begin{aligned}
& \tilde{\sigma}_{r r}^{*}=-\tilde{p}^{*}+2 \Lambda d_{r} \tilde{u}_{r}^{*} \\
& \tilde{\sigma}_{x r}^{*}=\Lambda\left(d_{r} \tilde{u}_{x}^{*}+i k^{*} \tilde{u}_{r}^{*}\right) .
\end{aligned}
$$

It is useful to note that the parameter $\Lambda$ represents the ratio of the elastic stresses in the wall material and the inertial or viscous stresses in the fluid. The limit $\Lambda \gg$ 1 represents the rigid wall limit, where the elasticity of the wall is large. In the complementary limit $\Lambda \ll 1$, the wall elasticity is small and this limit corresponds to a soft material which undergoes large deformation due to the flow.

The growth rate of the perturbations can now be obtained using the boundary conditions for the velocity and stress fields (8). The leading order boundary conditions, consistent with the magnitudes of the velocity, displacement and stress fields, equations $(25,26,28)$ are:

$$
\begin{aligned}
\tilde{v}_{w x}^{*}-2 \tilde{u}_{r}^{*} & =0, \\
\tilde{v}_{w r}^{*}+\tilde{v}_{o r}^{*} & =s^{*}, \\
\tilde{u}_{r}^{*}-\tilde{p}_{o}^{*} & =\tilde{\sigma}_{r r}^{*}, \\
\tilde{\sigma}_{x r}^{*} & =0 .
\end{aligned}
$$

The zero displacement conditions $\left(\tilde{u}_{r}^{*}=0\right.$ and $\left.\tilde{u}_{x}^{*}=0\right)$ at $r=H R$ and the shear stress condition $\tilde{\sigma}_{x r}^{*}=0$ at $r=0$ can be used to determine three of the constants, $B_{2}, B_{3}$ and $B_{4}$ as a function of $B_{1}$ in the solutions $(53,54)$ for the displacement fields. Using these, the ratio of the normal stress and the normal velocity at the unperturbed interface $r=R$ can be written as:

$$
\frac{\tilde{\sigma}_{r r}^{*}}{\tilde{u}_{r}^{*}}=-\Lambda C\left(k^{*}, H\right)
$$

where $C\left(k^{*}, H\right)$ is independent of the growth rate $s^{*}$, because the solutions for the displacement field $(53,54)$ do not depend on $s^{*}$. The constant $C\left(k^{*}, H\right)$ is always positive, and the product $C\left(k^{*}, H\right) \Lambda$ is the inverse of the "wall admittance" $Y$ defined by Landahl [15]. The use of (31) for the wall simplifies the calculations, since it is not necessary 


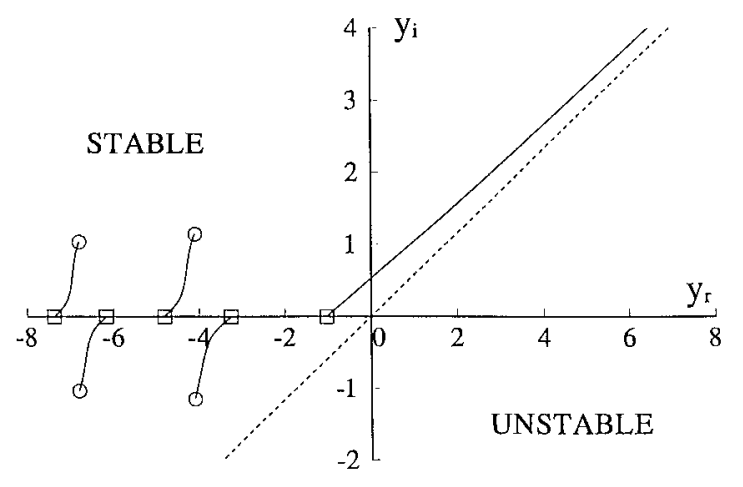

Fig. 2. Roots of the characteristic equation (32) for various values of $\Lambda^{*}$. The circles are the roots in the limit $\Lambda^{*} \rightarrow \infty$, and the squares are the roots in the limit $\Lambda^{*}=0$. The broken line shows the boundary between stable and unstable regimes.

to solve the equations for the fluid and the wall simultaneously in order to determine the growth rate. It should be noted that $C\left(k^{*}, H\right)$ is in general a complex quantity. However, in the asymptotic limit considered here, where the elastic term in the momentum equations for the wall displacement field is large compared to the viscous and acceleration terms, $C\left(k^{*}, H\right)$ is a real quantity.

Using (31) for $\left(\tilde{\sigma}_{r r}^{*} / \tilde{u}_{r}^{*}\right)$ and $(27)$ for $\left(\tilde{p}_{o}^{*} / \tilde{v}_{o r}^{*}\right)$, the velocity conditions in (30) can be simplified to give the characteristic equation for $y$ :

$$
i^{1 / 3} \Lambda^{*} \operatorname{Ai}(y, 1)-A i(y,-1)=0
$$

where $\Lambda^{*}=\left[\left(2 k^{*}\right)^{1 / 3} \Lambda C\left(k^{*}, H\right) / 4\right]$. The parameter $\Lambda^{*}$ has been introduced so that the solutions of (32) can be represented in terms of just one dimensionless parameter. This parameter is proportional to $\Lambda C\left(k^{*}, H\right)$, which is the ratio of the normal stress and the normal displacement in the wall material (see Eq. (31)). Since $C\left(k^{*}, H\right)$ is a positive function of $k^{*}$ and $H$, the coefficient $\Lambda^{*}$ can vary over the range $0 \leq \Lambda^{*} \leq \infty$. The limit $\Lambda^{*} \gg 1$ corresponds to the rigid wall case, while the limit $\Lambda^{*} \ll 1$ corresponds to the case of a soft material which undergoes large displacements due to stresses applied at the surface.

Equation (32) can be solved for $y$, and the growth rate $s^{*}=\left(2 i k^{*}\right)^{2 / 3} y$ at the interface $z^{*}=0$. The wall modes are stable for $(\pi / 2)<\operatorname{Arg}\left(s^{*}\right)<(3 \pi / 2)$, which corresponds to $(\pi / 6)<\operatorname{Arg}(y)<(7 \pi / 6)$ as shown by the broken line in Figure 2. It is useful to first examine the roots of the equation (32) in the limits $\Lambda^{*} \ll 1$ and $\Lambda^{*} \gg 1$. In the limit $\Lambda^{*} \gg 1\left(\left(G / \rho V^{2}\right) \gg \epsilon^{1 / 3}\right)$, the equation (32) reduces to:

$$
\tilde{v}_{w x}^{*}=A i(y, 1)=0 .
$$

The above equation is identical to that for the wall modes in a rigid tube [14]. In this limit, the velocity of the elastic surface is small compared to the fluid velocity in the wall layer, and the wall can be considered rigid in the leading approximation. There are multiple solutions for (33), all of which are stable. The four pairs of solutions having the lowest magnitudes are:

$$
\begin{aligned}
& y_{1}=-4.107 \pm 1.144 i \\
& y_{2}=-6.798 \pm 1.035 i \\
& y_{3}=-9.031 \pm 0.969 i \\
& y_{4}=-11.015 \pm 0.923 i
\end{aligned}
$$

The pair of solutions for $y_{1}$ is identical to that reported by Gill [14], but the other three differ slightly from Gill's results due to the use of an approximate formula by Gill for evaluating these solutions.

The limit $\Lambda^{*} \ll 1$ corresponds to $\left(G / \rho V^{2}\right) \ll \epsilon^{1 / 3}$. In this limit, the elastic stress in the wall material is small compared to the pressure in the fluid, and it is necessary to set the fluid pressure at the surface $\tilde{p}_{o}^{*}$ equal to zero in the leading approximation. In this limit, equation (32) reduces to:

$$
\operatorname{Ai}(y,-1)=0
$$

The roots of the above equation are on the negative real axis, as shown in Figure 2, and the roots that have the four lowest magnitudes are:

$$
\begin{aligned}
& y_{1}=-1.019, \\
& y_{2}=-3.248, \\
& y_{3}=-4.820, \\
& y_{4}=-6.163 .
\end{aligned}
$$

It can be seen form Figure 2 that the above roots are also stable.

Figure 2 also shows the trajectory of the roots as $\Lambda^{*}$ is varied. There is a smooth transition from the roots in the limit $\Lambda^{*} \ll 1(36)$ to the roots in the limit $\Lambda^{*} \gg 1(34)$. In addition, the root with the lowest magnitude, $y=-1.019$, in the limit $\Lambda^{*} \ll 1$ does not correspond to a continuation of any of the roots in the limit $\Lambda^{*} \gg 1$, but diverges in this limit. The reason for this behavior can be explained as follows. The asymptotic behavior of this root in the limit $\Lambda^{*} \gg 1$ can be determined from the large $y$ asymptotic expansion for the Airy functions in equation (32):

$$
\begin{aligned}
& \lim _{y \rightarrow \infty} A i(y, 1)=\exp \left[-(2 / 3) y^{3 / 2}\right] \\
& \quad \times\left(-0.282095 y^{-3 / 4}+0.240956 y^{-9 / 4}+O\left(y^{-15 / 4}\right)\right), \\
& \lim _{y \rightarrow \infty} A i(y,-1)=\exp \left[-(2 / 3) y^{3 / 2}\right] \\
& \quad \times\left(-0.282095 y^{1 / 4}-0.0411388 y^{-5 / 4}+O\left(y^{-11 / 4}\right)\right) .
\end{aligned}
$$

The ratio of these two terms is:

$$
\frac{A i(y,-1)}{A i(y, 1)}=y+\frac{1}{\sqrt{y}}+O\left(y^{-2}\right)
$$

Using the above expansion in (32), the limiting value of the divergent root is:

$$
y=i^{1 / 3} \Lambda^{*}-\left(i^{1 / 3} \Lambda^{*}\right)^{-1 / 2}+O\left(\Lambda^{*}\right)^{-2} .
$$


Using (24) for $y$ at the wall $\left(z^{*}=0\right)$, the growth rate is

$$
\begin{aligned}
s^{*}= & i(2 k)^{2 / 3} \Lambda^{*}-(2 k)^{2 / 3} \\
& \times\left(\frac{1+i}{\sqrt{2}}\right)\left(\Lambda^{*}\right)^{-1 / 2}+O\left(\Lambda^{*}\right)^{-2} .
\end{aligned}
$$

The above expression indicates that in the limit $\Lambda^{*} \gg 1$, there exists a root whose magnitude diverges proportional to $\Lambda^{*}$ which was not captured by the earlier $\Lambda^{*}=\infty$ analysis for a rigid tube [14]. Further, it can be seen that as $\Lambda^{*}$ increases, this root approaches the broken line in Figure 2 which represents the boundary between stable and unstable modes. This implies that this solution becomes less stable as the $\Lambda^{*}$ is increased, and the wall flexibility has a stabilizing effect on this solution. This solution, which is not a continuation of any of the rigid wall solutions is the least stable solution in a flexible tube. The frequency of this solution diverges proportional to $\Lambda^{*}$ and a decay rate that decreases proportional to $\left(\Lambda^{*}\right)^{-1 / 2}$ for $\Lambda^{*} \gg 1$. The physical reason for this can be explained as follows. Consider the case $\Lambda^{*} \gg 1$, where the normal stress $\tilde{\sigma}_{r r}^{*}$ increases proportional to $\Lambda^{*}$. From the boundary condition (30), it can be seen that the magnitude of the outer flow velocity $\tilde{v}_{o r}^{*}$ increases proportional to the frequency $\left|s^{*}\right|$ in the limit $\left|s^{*}\right| \gg 1$ while the tangential velocity $\tilde{v}_{w x}^{*}$ remains of the same magnitude as the normal displacement $\tilde{u}_{r}^{*}$. From the condition for the outer flow (27), this implies that there is an increase in the magnitude of the pressure proportional to $\left|s^{*}\right|$ as well. Thus, a balance between the normal stress in the wall and the pressure in the fluid is achieved when the magnitude of the frequency increases proportional to $\Lambda^{*}$, and the solution corresponding to this condition does not satisfy the zero normal velocity condition at the wall.

\section{Conclusions}

The growth rate of the wall modes in the limit of high Reynolds number was determined using asymptotic analysis. A scaling analysis showed that the viscous stresses in the fluid are of the same magnitude as the elastic stresses in the wall for $\Lambda=\epsilon^{-1 / 3}\left(G / \rho V^{2}\right) \sim 1$, where $G$ is the shear modulus of the surface, $V$ is the maximum velocity in the tube and $\epsilon=R e^{-1}$ is a small parameter. The linearized equations were solved using an expansion in the small parameter $\epsilon$. In the leading approximation, the velocity fluctuations are confined to a wall layer of thickness $O\left(\epsilon^{1 / 3}\right)$ smaller than the radius of the tube, and the velocity fluctuations in the outer region are $O\left(\epsilon^{1 / 3}\right)$ smaller than those in the wall layer. However, the pressure due to the outer inviscid flow turns out to be $O\left(\epsilon^{-1 / 3}\right)$ larger than the pressure and the normal stress in the wall layer, and is the dominant contribution to the normal stress in the fluid. The inertial and viscous effects in the wall are small compared to the elastic effects for $\Lambda \sim 1$, and the ratio of the normal stress and normal velocity in the wall is only a function of the wave number of the perturbation and the material parameters, and is independent of the frequency of the perturbations.
It is useful to compare the characteristics of the wall mode with those of the inviscid modes in a flexible tube $[6,7]$. In the inviscid modes, the leading order flow in the tube is inviscid, and the viscous dissipation does not influence the leading order frequency. The tangential velocity boundary condition requires the presence of a wall layer of thickness $O\left(\epsilon^{1 / 2}\right)$ at the wall. In the wall layer, there is a balance between the inertial term $\rho \partial_{t} v_{i}$ and the viscous term $\eta \partial_{j}^{2} v_{i}$, and the convective term $\rho v_{j} \partial_{j} v_{i}$ is small compared to the other terms. The presence of the wall layer results in a correction of $O\left(\epsilon^{1 / 2}\right)$ to the leading order frequency of fluctuations. In contrast, in the present case, the growth rate of the perturbations is $O\left(\epsilon^{1 / 3}\right)$ smaller than the leading order frequency. As a consequence, the leading order equations contain the inertial, convective and viscous term (see Eqs. $(22,21)$ ), and the leading order velocity fluctuation is confined to a wall layer of thickness $O\left(\epsilon^{1 / 3}\right)$ at the wall. The velocity due to the inviscid flow is $O\left(\epsilon^{1 / 3}\right)$ smaller than that in the wall layer, and the inviscid flow is driven by the flow in the wall layer.

In the asymptotic analysis, the decay rate of the perturbations depends on the wave number $k$ and the dimensionless number $\Lambda^{*} \equiv k^{* 1 / 3} \Lambda C\left(k^{*}, H\right)$, where $\left(-\Lambda C\left(k^{*}, H\right)\right)$ is the ratio of the normal stress and normal velocity in the wall material at the interface. In the limit $\Lambda^{*} \gg 0$, the elastic stress in the wall is large compared to the fluid pressure. The tangential velocity boundary conditions for the fluid reduces to a no-slip condition in this limit, and the wall resembles a rigid surface. The equation for the decay rate reduces to that obtained by Gill [14] for the flow through a rigid tube. There are multiple solutions for the decay rate, all of which have a negative real part, indicating that the fluctuations are stable in this limit. In the limit $\Lambda^{*} \ll 1$, the normal stress in the wall is small compared to that in the fluid, and the normal stress boundary condition for the fluid reduces to a zero normal stress condition. There are multiple solutions for the decay rate in this limit as well, and all these solutions have negative real parts, indicating that the fluctuations are stable. The solution for the decay rate makes a smooth transition between the two regimes. In addition, there is a non-trivial solution in the limit $\Lambda^{*} \ll 1$ which does not converge to any of the rigid wall solutions in the limit $\Lambda^{*} \gg 1$, but whose frequency increases proportional to $\Lambda^{*}$ and decay rate decreases proportional to $\Lambda^{*-1 / 2}$ in this limit. This solution, which is the least stable mode in a tube with large but finite elasticity, was not obtained in earlier studies of rigid walled tubes [14].

Though this new mode of oscillation does not go unstable in the asymptotic analysis, the asymptotic analysis fails as the parameter $\Lambda^{*}$ is increased, since the frequency of the perturbations increases proportional to $\Lambda^{*}$, and it is no longer justified to neglect the acceleration in the wall material. The earlier numerical studies $[8,9]$ on the continuation of the viscous modes, and a more recent numerical analysis [16] carried out specifically to probe the wall modes, show that a numerical continuation of this mode does become unstable as the Reynolds number is increased beyond a critical value for a given set of fluid and wall 
parameters. This explains why there are unstable modes in flexible channels and tubes with a boundary layer of thickness $O\left(R e^{-1 / 3}\right)$ smaller than the characteristic dimension. In addition, this mode is not a continuation of any of the rigid tube solutions, but is qualitatively different, and this is the reason why a similar instability is not observed in a rigid tube, where the wall modes (asymptotic and numerical) are always stable.

\section{Appendix A}

The solution for the components of the wall layer velocity $\tilde{v}_{w x}^{*}$ and $\tilde{v}_{w r}^{*}$ and the pressure $\tilde{p}_{w}^{*}$ are obtained as follows from the equations $(19,21,22)$. An equation for the axial velocity in the wall layer is obtained taking the derivative of (21) with respect to $z^{*}$, and using (19) to express $\tilde{v}_{w r}^{*}$ in terms of $\tilde{v}_{w x}^{*}$ :

$$
\left[-\left(s^{*}+2 i k^{*} z^{*}\right)+d_{z^{*}}^{2}\right] d_{z^{*}} \tilde{v}_{w x}^{*}=0 .
$$

The above third order differential equation for $\tilde{v}_{w x}^{*}$ has the solution:

$$
\tilde{v}_{w x}^{*}=C_{1}+C_{2} A i(y, 1)+C_{3} B i(y, 1),
$$

where

$$
y=\left(2 i k^{*}\right)^{1 / 3}\left[\left(s^{*} / 2 i k^{*}\right)+z^{*}\right],
$$

$A i(y)$ and $B i(y)$ are the Airy functions which are solutions of the equation $\left(d_{y}^{2}+y\right) \psi(y)=0$, and the generalized Airy functions are given by (Drazin and Reid [10]):

$$
\begin{aligned}
& A i(y, 1)=\int_{\infty}^{y} d y \operatorname{Ai}(y), \quad A i(y,-1)=\frac{d A i(y)}{d y}, \\
& B i(y, 1)=\int_{\infty}^{y} d y \operatorname{Bi}(y), \quad B i(y,-1)=\frac{d B i(y)}{d y} .
\end{aligned}
$$

The coefficients $C_{1}, C_{2}$ and $C_{3}$ in (42) are determined by matching the solution for the tangential velocity in the wall layer with that in the outer region. It is shown a little later that the tangential velocity in the outer region scales as $\epsilon^{1 / 3} \tilde{v}_{w x}$, and the matching condition requires that $\tilde{v}_{w x}^{*} \rightarrow 0$ for $z^{*} \rightarrow \infty$. The Airy functions $A i(y, p)$ are convergent in the limit $y \rightarrow \infty$ only for $(-\pi / 3)<\operatorname{Arg}(y)<(\pi / 3)$, and so it is necessary to choose $\operatorname{Arg}\left(i^{1 / 3}\right)=(\pi / 6)$ in (24). In this domain, the Airy function $B i(y, p)$ diverges, and so the matching condition requires that $C_{1}=0$ and $C_{3}=0$ in (42). With this, the solutions for the velocity profiles and the pressure in the wall layer are:

$$
\begin{aligned}
\tilde{v}_{w x}^{*} & =A i(y, 1), \\
\tilde{v}_{w r}^{*} & =2^{-1 / 3}\left(i k^{*}\right)^{2 / 3}[y A i(y, 1)-A i(y,-1)], \\
\tilde{p}_{w}^{*} & =0 .
\end{aligned}
$$

The coefficient $C_{2}$ in (42) has been set equal to 1 without loss of generality. The expression for the tangential velocity in (46) is identical to that obtained by Gill [14] for the flow in a rigid tube.

\section{Appendix B}

The linearized equations of motion for the wall material (from Eqs. $(5,6)$ ) are:

$$
\left(d_{r^{*}}+r^{*-1}\right) \tilde{u}_{r}+i k^{*} \tilde{u}_{x}=0
$$

$$
\begin{aligned}
& -d_{r^{*}} \tilde{p}+\left[-\rho \epsilon^{2 / 3} s^{* 2}+\left(G+\epsilon^{1 / 3} s^{*} \eta_{s}\right)\right. \\
& \left.\times\left(d_{r^{*}}^{2}+r^{*-1} d_{r^{*}}-r^{*-2}-k^{* 2}\right)\right] \tilde{u}_{r}=0 \\
& -i k^{*} \tilde{p}+\left[-\rho \epsilon^{2 / 3} s^{* 2}+\left(G+\epsilon^{1 / 3} s^{*} \eta_{s}\right)\right. \\
& \left.\times\left(d_{r^{*}}^{2}+r^{*-1} d_{r^{*}}-k^{* 2}\right)\right] \tilde{u}_{x}=0
\end{aligned}
$$

where $r^{*}=(r / R)$. Consistent with the magnitudes (28), the scaled displacement and pressure fields in the elastic medium are defined as $\tilde{u}_{i}^{*}=\left(\tilde{u}_{i} / R\right)$ and $\tilde{p}^{*}=\tilde{p} /\left(\epsilon^{1 / 3} \rho V^{2}\right)$ to obtain the following dimensionless equations:

$$
\left(d_{r^{*}}+r^{*-1}\right) \tilde{u}_{r}^{*}+i k^{*} \tilde{u}_{x}^{*}=0
$$

$$
\begin{aligned}
-d_{r^{*}} \tilde{p}^{*}+[ & -\epsilon^{1 / 3} s^{* 2}+\left(\Lambda+\epsilon s^{*} \eta_{r}\right) \\
& \left.\times\left(d_{r^{*}}^{2}+r^{*-1} d_{r^{*}}-r^{*-2}-k^{* 2}\right)\right] \tilde{u}_{r}^{*}=0
\end{aligned}
$$

$$
\begin{aligned}
-i k^{*} \tilde{p}^{*}+\left[-\epsilon^{1 / 3} s^{* 2}\right. & +\left(\Lambda+\epsilon s^{*} \eta_{r}\right) \\
& \times\left(d_{r^{*}}^{2}+r^{*-1} d_{r^{*}}-k^{* 2}\right] \tilde{u}_{x}^{*}=0
\end{aligned}
$$

where $\eta_{r}=\left(\eta_{s} / \eta\right)$ is the ratio of the viscosities of the fluid and the wall material. In the leading order approximation, the acceleration term proportional to $\epsilon^{1 / 3}$ and the gradient of the viscous stress proportional to $\epsilon \eta_{r}$ can be neglected, and the solutions for the displacement fields are:

$$
\begin{aligned}
\tilde{u}_{r}^{*}= & B_{1} r^{*} K_{0}\left(k^{*} r^{*}\right)+B_{2} K_{1}\left(k^{*} r^{*}\right)+B_{3} r^{*} I_{0}\left(k^{*} r^{*}\right) \\
& +B_{4} I_{1}\left(k^{*} r^{*}\right) \\
\tilde{u}_{x}^{*}= & \left(i B_{1} / k^{*}\right)\left[2 K_{0}\left(k^{*} r^{*}\right)-k^{*} r^{*} K_{1}\left(k^{*} r^{*}\right)\right] \\
& -i B_{2} K_{0}\left(k^{*} r^{*}\right)+\left(i B_{3} / k^{*}\right)\left[2 I_{0}\left(k^{*} r^{*}\right)\right. \\
& \left.+k^{*} r^{*} I_{1}\left(k^{*} r^{*}\right)\right]+i B_{4} I_{0}\left(k^{*} r^{*}\right) \\
\tilde{p}^{*}= & 2 \Lambda\left[B_{1} K_{0}\left(k^{*} r^{*}\right)+B_{3} I_{0}\left(k^{*} r^{*}\right)\right]
\end{aligned}
$$

where $I_{0}\left(k^{*} r^{*}\right), I_{1}\left(k^{*} r^{*}\right),\left(K_{0}\left(k^{*} r^{*}\right)\right)$ and $\left(K_{1}\left(k^{*} r^{*}\right)\right)$ are modified Bessel functions, and $\left(B_{1}\right),\left(B_{2}\right),\left(B_{3}\right)$ and $\left(B_{4}\right)$ are constants to be determined from the boundary conditions. Two of these are fixed by the zero displacement conditions $\left(\left(\tilde{u}_{r}^{*}=0\right)\right.$ and $\left.\left(\tilde{u}_{x}^{*}=0\right)\right)$ at $\left(r^{*}=H\right)$, while the other two are determined from the conditions at the interface. 
The author would like to thank the Department of Science and Technology, Government of India for financial support.

\section{References}

1. P. Krindel, A. Silberberg, J. Coll. Interf. Sci. 71, 34 (1979).

2. R. Bar-Ziv, E. Moses, Phys. Rev. Lett. 73, 1392 (1994).

3. P. Nelson, T. Powers, U. Seifert, Phys. Rev. Lett. 74, 3384 (1995).

4. V. Kumaran, G.H. Fredrickson, P. Pincus, J. Phys. II France 4, 893 (1994).

5. V. Kumaran, J. Fluid Mech. 294, 259 (1995).
6. V. Kumaran, J. Chem. Phys. 102, 3452 (1995).

7. V. Kumaran, J. Fluid Mech. 302, 117 (1995).

8. L. Srivatsan, V. Kumaran, J. Phys. II France 7, 947 (1997).

9. V. Kumaran, J. Fluid Mech. 357, 123 (1998).

10. P.G. Drazin, W.H. Reid, Hydrodynamic Stability (Cambridge University Press, 1981).

11. L.D. Landau, E.M. Lifshitz, Theory of Elasticity (Pergamon, New York, 1989).

12. J.H. Harden, H. Pleiner, P.A. Pincus, J. Chem. Phys. 94, 5208 (1991).

13. V. Kumaran, J. Chem. Phys. 98, 3429 (1993).

14. A.E. Gill, J. Fluid Mech. 21, 145 (1965).

15. M.T. Landahl, J. Fluid Mech. 13, 609 (1962).

16. V. Kumaran, J. Fluid Mech. (in press). 